\title{
Providing Automatic Support for Heuristic Rules of Methods
}

\author{
Bedir Tekinerdogan \& Mehmet Aksit \\ TRESE project, Department of Computer Science, \\ University of Twente, P.O. Box 217, 7500 AE Enschede, The Netherlands. \\ email: \{bedir|aksit\}@cs.utwente.nl,www server: http://wwwtrese.cs.utwente.nl
}

\begin{abstract}
In method-based software development, software engineers create artifacts based on the heuristic rules of the adopted method. Most CASE tools, however, do not actively assist software engineers in applying the heuristic rules. To provide an active support, the rules must be formalized, implemented and integrated within the framework of current CASE tools. In this paper we describe an approach for formalizing the heuristic rules of methods.
\end{abstract}

\section{Introduction}

It is generally agreed that the success of a software development method largely depends on the availability of tools supporting it. In general, a software development method can be defined in terms of three aspects: representation models, software process and method rules [Budgen 93]. Representation models are descriptive forms that the software engineer can utilize for building models. A software process is a description of the order of the phases of a method. Method rules aim at identifying, eliminating and verifying the artifacts. A considerable number of CASE environments have been developed during the past years. Most CASE environments provide tools for definition of artifacts and processes. Very few of them, however, provide support for the application of rules of methods. The lack to capture and support the heuristic rules of methods reduces the effectiveness of current CASE environments.

In this paper we provide a generic approach for formalizing and implementing heuristic rules of methods. We defined a knowledge acquisition technique to extract the rules of a method. In this approach, rules are expressed using production-rules. We developed an agent-based reasoning system to integrate the rules with the method artifacts.

\section{Heuristics of Methods}

Most methods define rules in an informal manner. Nevertheless, method rules can be expressed using conditional statements in the form IF <condition> THEN 
<consequent>. The consequent part may be an action. For example, the method OMT [Rumbaugh 91] advises the following rule to identify classes:

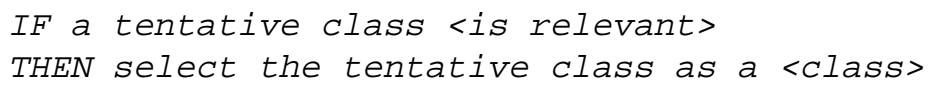

Compared to the research activities on representation models and software processes, there has been very little effort in formalizing design heuristics. In the literature [Rumbaugh 91][Riel 96] object-oriented design heuristics are generally explained in textual form.

In the area of expert system design, representation of heuristic rules have been extensively studied [Gonzales 93]. For example, in the area of mechanical and electronic engineering, a number of expert systems have been developed to represent various kinds of expert's knowledge. In general, these systems are integrated into a CAD environment. To the best of our knowledge, there has not been any commercial CASE environment, which provides an integrated expert system support to guide software engineers actively in applying heuristic rules. Therefore, we think that it is worthwhile to investigate the possibility of using an expert system support for CASE environments.

\section{Heuristic Rule Modeling}

Our attempt to formalize software development methods is a special case of knowledge acquisition as described in traditional knowledge engineering techniques [Gonzales 93]. The domain for knowledge engineering hereby is the software development method for which we would like to define CASE support. We acquire our knowledge mainly by studying the available documentation on the methods and by interviewing experts of the corresponding methods [Hart 92]. Fig. 1 represents the overview for this heuristic method rule acquisition process.

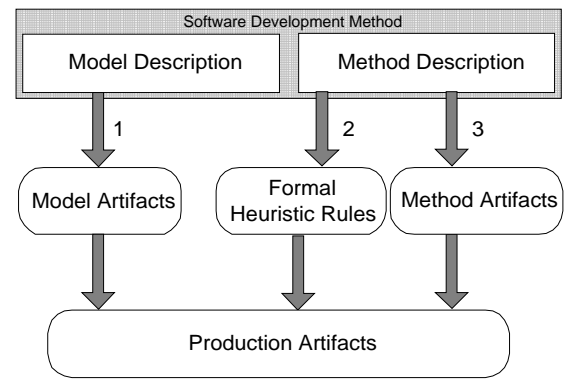

Fig. 1 Heuristic Method Rule acquisition

Each method can be considered as having a model description and method description. In the model description the representation models are described. The method description part describes the process and the informal heuristic rules for instantiating these representation models. In OMT the model description part describes for example the representation model class. In the method description part heuristic 
rules for identification of classes are described. The method description may use additional representation models, which are not described in the model description. These may be graphical diagrams or temporary representation models like the tentative class in the OMT class identification process.

The method formalization process includes basically four steps. First the model artifacts are extracted from the model description. Second the informal heuristic rules from the method description are identified and formalized. Third, from the heuristic rules method artifacts are derived. Fourth, the formalized rules are assigned to the model and method artifacts.

\section{Conclusions}

In this paper we defined a knowledge modeling method for formalizing the heuristic rules of methods. The approach is aimed to be used for a wide range of wellestablished methods and for integration with existing CASE tools. For illustration purposes we applied our approach to the analysis phase of OMT and formalized the heuristic rules of it. We are experimenting with the development of a CASE tool, which we are integrating with the Rational Rose tool. We experienced that our experimental CASE tool helped the software engineers in applying heuristic rules in designing their systems.

The problems that we encountered in representing the heuristic rules can be grouped as problems related to the method being formalized and the problems related to the adopted knowledge representation and reasoning technique.

\section{References}

1. [Budgen 93] D. Budgen, Software Design, Addison-Wesley, 1994.

2. [Gonzales 93] A.J. Gonzales, \& D.D. Dankel, The Engineering of Knowledge-Based Systems, Prentice Hall, 1993.

3. [Hart 92] A. Hart, Knowledge acquisition for expert systems, McGraw-Hill, 1992.

4. [Rumbaugh 91] J. Rumbaugh, M. Blaha, W. Premerlani, F. Eddy, \& W. Lorensen, ObjectOriented Modeling and Design, Prentice-Hall, 1991.

5. [Riel 96] A. Riel. Object Oriented Design Heuristics, Addison-Wesley, 1996 